ARQGA / 926

\title{
ESTUDO DO ESVAZIAMENTO GÁSTRICO E DA DISTRIBUIÇÃO INTRAGÁSTRICA DE UMA DIETA SÓLIDA ATRAVÉS DA CINTILOGRAFIA:
}

\section{diferenças entre os sexos}

\begin{abstract}
Sônia Letícia Silva LORENA*, Eduardo TINOIS**, Eunice Sizue HIRATA***, Marcelo Livorsi CUNHA**, Sérgio Quirino BRUNETTO**, Edwaldo Eduardo CAMARGO** e Maria Aparecida MESQUITA*
\end{abstract}

\begin{abstract}
RESUMO - Ainda existem controvérsias quanto a possiveis diferenças de esvaziamento gástrico entre os sexos. Os objetivos deste estudo são: confirmar se homens e mulheres realmente diferem no esvaziamento gástrico de uma dieta sólida e investigar se esta diferença está relacionada com diferentes padrões de distribuição da dieta entre os compartimentos proximal e distal do estômago. Dezoito voluntários assintomáticos (nove do sexo masculino, com idade média de $35 \pm 9$ anos e nove do sexo feminino, com idade média de $41 \pm 11$ anos) foram estudados pela manhã, após ingestão da dieta sólida padronizada (omelete marcada com $185 \mathrm{MBq}$ de enxofre coloidal- ${ }^{99 m} \mathrm{Tc}$ ). Imagens simultâneas do estômago nas projeções anterior e posterior foram adquiridas imediatamente após a ingestão da dieta e a cada 10 minutos, durante 120 minutos. Curvas de tempo versus contagem radioativa foram obtidas para o estômago total, proximal e distal. O T112 do esvaziamento gástrico foi maior para as mulheres (96,1 $\pm 17,2$ minutos) do que para os homens (79,9 $\pm 17,8$ minutos; $P$ =0,02). A análise da distribuição intragástrica da dieta mostrou que não houve diferenças entre os sexos quanto ao esvaziamento gástrico proximal, mas as mulheres apresentaram maior retenção da dieta na porção distal do estômago $(P=0,04)$. Nossos resultados mostram que o esvaziamento gástrico de sólidos é mais lento nas mulheres não-menopausadas assintomáticas, do que nos homens de faixa etária semelhante, provavelmente devido a maior retenção da dieta na região distal do estômago. Este fato deve ser levado em conta nos estudos de esvaziamento gástrico pela cintilografia para evitar diagnósticos errôneos de gastroparesias nas pacientes do sexo feminino.
\end{abstract}

DESCRITORES - Esvaziamento gástrico. Sexo. Cintilografia.

Disciplina de Gastroenterologia, Departamento de Clínica Médica, Faculdade de Ciências Médicas de Campinas - FCM-UNICAMP, Campinas, SP.

** Serviço de Medicina Nuclear, Departamento de Radiologia, FCM-UNICAMP.

*** Departamento de Anestesiologia, FCM-UNICAMP.

Endereço para correspondência: Dra. Maria Aparecida Mesquita - Disciplina de Gastroenterologia - Departamento de Clínica Médica da Faculdade de Ciências

Médicas da UNICAMP - C.P. 6111 - 13081-970 - Campinas, SP. e-mail: mesquita@fcm.unicamp.br 


\section{INTRODUÇÃO}

O estudo do esvaziamento gástrico por cintilografia é considerado atualmente o padrão-ouro para a avaliação do trânsito gástrico. Recentemente, a complementação dos estudos com a análise da distribuição da dieta nos compartimentos proximal e distal do estômago, associada à aplicação de métodos mais refinados de análise matemática das curvas de esvaziamento gástrico, trouxe avanços significativos na interpretação dos exames, abrindo novas perspectivas para melhor conhecimento tanto da fisiologia motora, quanto da fisiopatologia dos distúrbios da motilidade gástrica ${ }^{(14)}$.

Embora a técnica já esteja bastante difundida, observa-se na literatura que ainda existem controvérsias quanto aos parâmetros de normalidade dos estudos, especialmente no que se refere às possíveis diferenças entre os sexos masculino e feminino. Enquanto alguns autores não observaram influência do sexo no esvaziamento gástrico ${ }^{(4,5,10,11,13)}$, estudos mais recentes demonstram que o sexo feminino apresenta esvaziamento gástrico mais lento ${ }^{(1,2,3,7,8)}$.

Sabe-se que grande parte dos pacientes com queixas sugestivas de gastroparesia é do sexo feminino ${ }^{(12)}$. Entretanto, freqüentemente os valores de normalidade dos exames de esvaziamento gástrico são obtidos através do estudo de grupos-controle constituídos predominantemente por voluntários do sexo masculino, ou por proporções variadas de homens e mulheres. Caso o trânsito gástrico seja realmente diferente entre homens e mulheres, estas diferenças deveriam ser obrigatoriamente consideradas quando da interpretação dos exames.

Os objetivos deste estudo são, portanto: 1) Confirmar a existência de diferenças no esvaziamento gástrico de uma dieta sólida entre voluntários assintomáticos do sexo feminino e masculino. 2) Avaliar se estas diferenças estão associadas a diferentes padrões de distribuição da dieta entre as regiões proximal e distal do estômago.

\section{SUJEITOS E MÉTODOS}

Foram estudados 18 voluntários assintomáticos: nove do sexo masculino (idade média: $35 \pm 9$ anos) e nove do feminino (idade média: $41 \pm 11$ anos), recrutados entre funcionários e médicos do Hospital das Clínicas da Universidade Estadual de Campinas HCUNICAMP. Nenhum participante era obeso, tinha história de doença sistêmica, doença gastrointestinal, cirurgia prévia, ou estava em uso de medicações que pudessem afetar a motilidade gastrointestinal. Nenhuma das mulheres estava na menopausa, sendo que todos os estudos foram realizados durante a primeira fase do ciclo menstrual. O estudo foi aprovado pela Comissão de Ética do HC-UNICAMP.

\section{Dieta-teste}

A dieta-teste foi uma omelete feita com três ovos misturados com $185 \mathrm{MBq}$ de enxofre coloidal- ${ }^{99 \mathrm{~m}} \mathrm{Tc}$, cozida em forno microondas até adquirir uma consistência firme, e ingerida com $100 \mathrm{~mL}$ de água, em 5 minutos.

\section{Estudo do esvaziamento gástrico}

Os participantes foram estudados pela manhã, após jejum de pelo menos 10 horas. Após ingestão da dieta-teste, sentavam-se em uma cadeira colocada entre as duas cabeças da câmara de cintilação (Sopha DST), equipadas com colimadores de baixa energia e uso genérico. A região epigástrica foi colocada no centro do campo de visão das cabeças anterior e posterior. Imagens simultâneas das regiões anterior e posterior do estômago foram adquiridas por 60 segundos imediatamente após a ingestão da dieta e a cada 10 minutos, por 120 minutos.

Para o processamento das imagens, foi determinada a área gástrica de interesse (ROI) nas projeções anterior e posterior de cada imagem adquirida. Após correção para decaimento radioativo, para cada aquisição foi determinada a média geométrica das contagens radioativas da região anterior e posterior, e os valores foram expressos como porcentagem da maior contagem. Com estes dados foram obtidas curvas de tempo versus contagem radioativa.

\section{Distribuição da dieta entre os compartimentos proximal e distal do estômago}

Para a análise separada das curvas de esvaziamento gástrico proximal e distal, o estômago foi dividido nas duas regiões através de uma linha reta passando na região da incisura angularis. Para cada aquisição, as médias geométricas das contagens radioativas nas projeções anterior e posterior foram expressas como porcentagem da maior contagem encontrada no estômago total, obtendo-se, então, curvas de tempo versus porcentagem das contagens radioativas para os dois compartimentos gástricos.

\section{Análise dos resultados}

Para cada estudo, foram determinados os seguintes parâmetros: 1) $\mathrm{T}^{1} \frac{1}{2}$ do esvaziamento gástrico total, isto é, o tempo para que $50 \%$ da dieta tivesse deixado o estômago; 2) $\mathrm{T}^{1} \frac{1}{2}$ do esvaziamento proximal; 3) Máximo conteúdo distal, definido como a maior porcentagem da atividade radioativa encontrada na região distal em qualquer período do estudo; e 4) Área sob a curva de retenção gástrica distal.

\section{Análise estatística}

Os resultados foram expressos como média \pm desvio padrão, e comparados através do teste de Mann-Whitney. Valores de $P$ menores que 0,05 foram considerados como estatisticamente significativos. 


\section{RESULTADOS}

\section{Esvaziamento gástrico}

A Figura 1A apresenta as curvas médias de esvaziamento gástrico para os voluntários dos dois sexos. Nota-se que as mulheres apresentaram maior retenção gástrica do alimento durante o estudo, indicando que o esvaziamento gástrico foi mais prolongado no sexo feminino. O T $1 \frac{1}{2}$ do esvaziamento gástrico de $96,1 \pm 17,2$ minutos no sexo feminino foi significativamente mais alto que o $\mathrm{T}^{1} 1 / 2$ de 79,9 $\pm 17,8$ minutos encontrado no sexo masculino (Tabela 1 ).

TABELA 1 - Comparação do esvaziamento gástrico e da distribuição da dieta entre as regiões proximal e distal do estômago entre os sexos feminino e masculino

\begin{tabular}{lccc}
\hline Parâmetros & Sexo Feminino & Sexo Masculino & \multicolumn{1}{c}{$\boldsymbol{P}$} \\
\hline $\mathrm{T}^{1} / 2(\min )$ & $96,1 \pm 17,2$ & $79,9 \pm 17,8$ & $0,02^{*}$ \\
$\mathrm{~T} 1 / 2$ proximal $(\min )$ & $34 \pm 22$ & $34,2 \pm 14,5$ & 0,98 \\
ASC- distal $\left(\mathrm{x} 10^{-4}\right)$ & $20,4 \pm 6,5$ & $14,9 \pm 5,5$ & $0,04^{*}$ \\
CD Máximo $(\%)$ & $0,39 \pm 0,11$ & $0,31 \pm 0,12$ & 0,22 \\
\hline
\end{tabular}

$* P<0,05$

ASC-distal: média dos valores da área sob a curva de retenção distal na fase de esvaziamento

CD: conteúdo distal

\section{Distribuição intragástrica da dieta}

As Figuras 1B e 1C mostram as respectivas curvas médias de retenção gástrica nos compartimentos proximal e distal do estômago para os dois sexos. Na Tabela 1 encontram-se os parâmetros utilizados para a comparação da distribuição intragástrica entre os sexos.

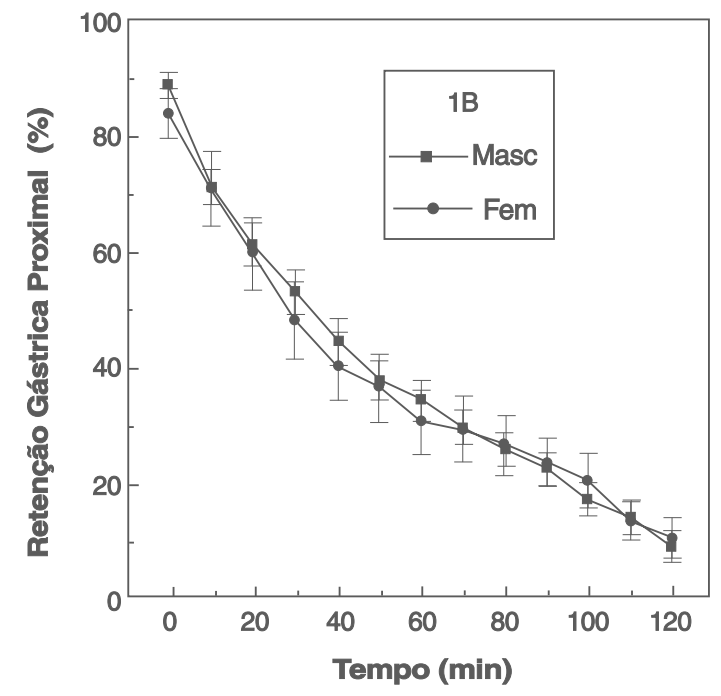

FIGURA 1 - Diferenças entre os sexos no esvaziamento gástrico de uma dieta sólida. Curvas médias de retenção gástrica no: 1A: estômago total; 1B: estômago proximal e 1C: estômago distal. $\mathrm{O}$ esvaziamento gástrico foi mais lento entre as mulheres, que também apresentaram maior retenção da dieta na região distal do estômago.
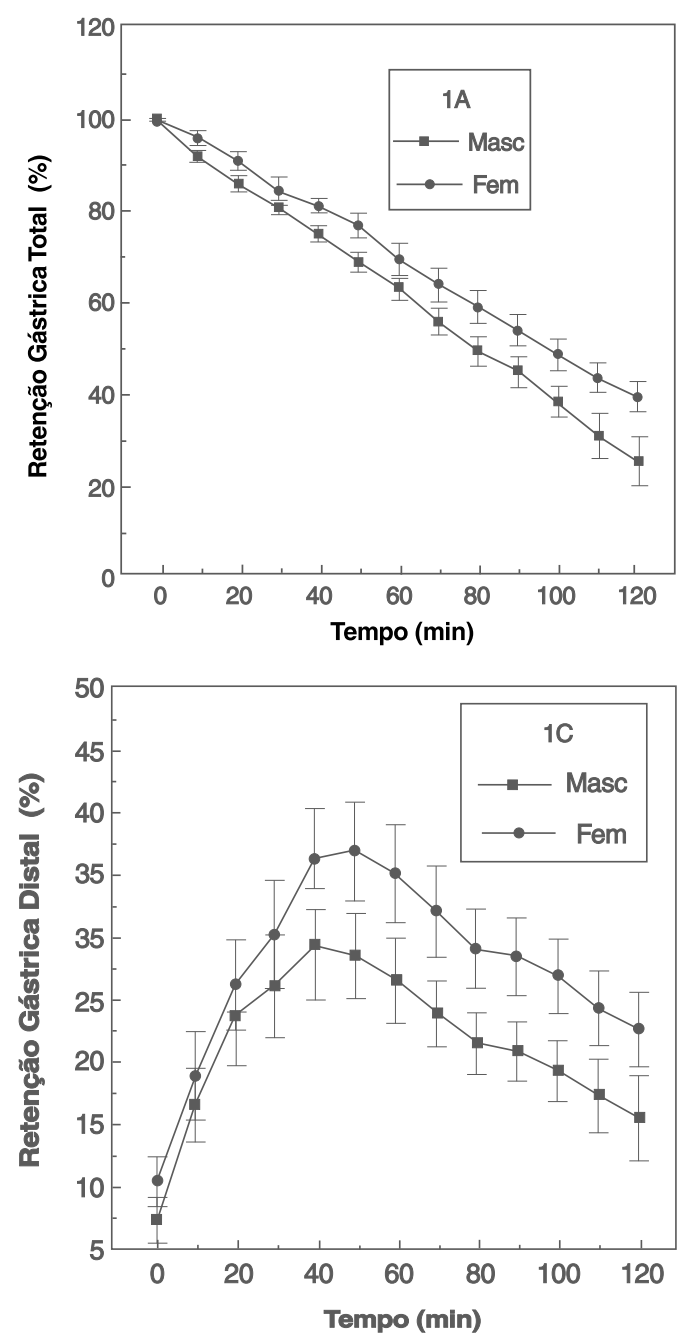
Observam-se que as curvas de retenção gástrica proximal (Figura 1B) de homens e mulheres são muito semelhantes, indicando que o esvaziamento da dieta no compartimento proximal do estômago foi similar para os dois sexos. Não houve diferença estatisticamente significativa entre homens e mulheres quanto aos valores do $\mathrm{T}^{1} / 2$ do estômago proximal.

A curva de retenção gástrica distal (Figura 1C) apresenta duas fases: inicialmente, o conteúdo radioativo vai aumentando até um valor máximo de aproximadamente 30 a $40 \%$ das contagens radioativas totais, atingido em torno de 50 minutos após a ingestão da dieta na maior parte dos voluntários de ambos os sexos. Após este período, inicia-se a fase de esvaziamento do antro. A comparação das curvas de retenção distal dos sexos masculino e feminino mostra que na fase de esvaziamento do antro, os valores da área sob a curva foram significativamente mais altos para o sexo feminino, indicando maior retenção da dieta no compartimento distal do estômago deste grupo. Não houve diferença estatisticamente significativa entre os dois sexos quanto ao conteúdo máximo das contagens na região distal do estômago, embora os resultados também apontem para uma tendência a valores superiores entre as mulheres.

\section{DISCUSSÃO}

Nossos resultados demonstram que voluntárias assintomáticas do sexo feminino apresentam esvaziamento gástrico de sólidos mais lento que o observado entre voluntários do sexo masculino.

A existência de diferenças entre os sexos no esvaziamento gástrico tem sido objeto de controvérsias. Entretanto, nos últimos 10 anos vários estudos têm trazido evidências de que o sexo feminino apresenta trânsito gástrico mais lento não só de dietas sólidas, como possivelmente também de dietas líquidas ${ }^{(1,3)}$.

A comparação entre controles do sexo feminino nas fases pré e pós-menopausa sugere que o prolongamento do trânsito gástrico seria conseqüência da influência hormonal ${ }^{(7)}$. Mulheres na fase da menopausa sem reposição hormonal apresentam trânsito gástrico semelhante ao observado no sexo masculino. Em contraste, aquelas recebendo tratamento hormonal apresentam esvaziamento gástrico mais lento que os homens. Foi demonstrado que os hormônios sexuais femininos teriam uma atuação direta sobre a musculatura gastrointestinal, diminuindo a sua contratilidade ${ }^{(9)}$.

Considerando a provável influência hormonal sobre o esvaziamento gástrico, muitos autores preconizaram que os estudos fossem realizados apenas na primeira fase do ciclo menstrual, quando os níveis de progesterona e outros hormônios estariam mais baixos ${ }^{(15)}$. Entretanto, outros autores observaram que as diferenças entre os dois sexos persistiam, mesmo quando era adotado o procedimento de realizar os exames apenas na fase folicular do ciclo menstrual ${ }^{(8)}$. Nossos resultados confirmam este último estudo, uma vez que as participantes do sexo feminino de nosso grupo foram estudadas na primeira fase do ciclo menstrual.

A análise das curvas de retenção gástrica nos compartimentos proximal e distal do estômago demonstrou que o esvaziamento mais lento da dieta sólida em nossas voluntárias do sexo feminino associouse a maior retenção do alimento no compartimento distal do estômago. Sabe-se que o esvaziamento gástrico depende da ação coordenada entre o estômago proximal, antro, piloro e duodeno ${ }^{(6)}$. Neste estudo, não houve diferenças entre os sexos quanto ao esvaziamento gástrico proximal, o que sugere que a função motora deste compartimento, incluindo a acomodação, seja semelhante para homens e mulheres. A maior retenção da dieta no antro poderia, então, originar-se de diferenças na motilidade do antro ou da região piloroduodenal. Não existem estudos avaliando um possível efeito do sexo na motilidade piloroduodenal. Os estudos que investigaram o efeito do sexo sobre a motilidade do antro gástrico apresentaram resultados discordantes. Estudos de manometria mostraram que homens e mulheres apresentam motilidade antral semelhante ${ }^{(7)}$, ou que a contratilidade antral é menor no sexo feminino ${ }^{(8)}$. Mais recentemente, o estudo da motilidade do antro através da cintilografia dinâmica, também tem mostrado resultados divergentes ${ }^{(1,8)}$

Em resumo, este estudo demonstrou que mulheres nãomenopausadas apresentam esvaziamento gástrico de sólidos mais lento que homens de faixa etária semelhante, e que esta diferença está relacionada a maior retenção do alimento no compartimento distal do estômago das mulheres. Estas particularidades devem ser consideradas quando da interpretação dos exames de pacientes com gastroparesias ou com dispepsia funcional, que em grande parte são do sexo feminino. Valores de normalidade derivados de grupos-controle mistos ou predominantemente masculinos podem levar a diagnósticos errôneos de gastroparesias, quando as anormalidades são simplesmente variações normais relacionadas ao sexo. Como existem evidências na literatura de que as diferenças entre os sexos podem manter-se mesmo quando os exames são realizados na primeira fase do ciclo menstrual, o que foi confirmado por nosso estudo, é recomendável que os valores de normalidade dos estudos de esvaziamento gástrico sejam diferenciados para cada sexo. 
Lorena SLS, Tinois E, Hirata ES, Cunha ML, Brunetto SQ, Camargo EE, Mesquita MA. Scintigraphic study of gastric emptying and intragastric distribution of a solid meal: gender differences. Arq Gastroenterol 2000;37(2):102-106.

ABSTRACT - Gender-related differences in gastric emptying are still controversial. The aims of this study were: to confirm the sex-related difference in gastric emptying of a solid meal and to investigate its association with different patterns of meal distribution between the proximal and distal gastric compartments. Eighteen healthy volunteers (nine males, mean age $35 \pm 9$ years; nine females, mean age: $41 \pm$ 11 years) were studied in the morning, after ingestion of the solid test-meal (an omelette labeled with $185 \mathrm{MBq}$ of ${ }^{99 \mathrm{~m}} \mathrm{Tc}$-sulfur colloid). Simultaneous anterior and posterior images of the stomach were acquired immediately after ingestion of the meal and every 10 minutes for 120 minutes. Time versus activity curves were obtained for the whole, proximal and distal stomach. Gastric T1/2 was longer in women (96.1 $\pm 17.2 \mathrm{~min})$ than in men $(79.9 \pm 17.8 \mathrm{~min} ; P=0.02)$. The analysis of the meal distribution inside the stomach showed no differences between males and females in proximal gastric emptying, but the meal retention in the distal compartment was significantly increased among women $(P=0.04)$. In conclusion, gastric emptying of a solid meal is slower in pre-menopausal women than in age-matched men, probably due to an increased retention of the meal in the distal compartment. This should be taken into consideration to avoid misleading diagnosis of gastroparesis for female patients.

HEADINGS - Gastric emptying. Gender. Scintigraphy.

\section{REFERÊNCIAS BIBLIOGRÁFICAS}

1. Bennink R, Peeters M, Van den Maegdenbergh V, Geypens B, Rutgeerts P, de Roo M, Mortelmans L. Comparison of total and compartmental gastric emptying and antral motility between healthy men and women. Eur J Nucl Med 1998;25:1293-9.

2. Gill RC, Murphy PD, Hooper HR. Effect of menstrual cycle on gastric emptying. Digestion 1987;36:168-74.

3. Gryback P, Naslund E, Hellstrom PM, Jacobsson H, Backman L. Gastric emptying of solids in humans: improved evaluation by Kaplan-Meyer plots, with special reference to obesity and gender. Eur J Nucl Med 1996;23:1562-7.

4. Horowitz M, Maddern GJ, Chatterton BE, Collins PJ, Harding PE, Shearman DJC. Changes in gastric emptying rates with age. Clin Sci 1984;67:213-8.

5. Horowitz M, Maddern GJ, Chatterton BE. The normal menstrual cycle has no effect on gastric emptying. Br J Obstet Gynecol 1985;92:743-6.

6. Horowitz M, Dent J, Fraser R, Sun W, Hebbard G. Role and integration of mechanisms controlling gastric emptying. Dig Dis Sci 1994;39 Suppl:7S-13S.

7. Hutson WR, Roehrkasse RL, Wald A. Influence of gender and menopause on gastric emptying and motility. Gastroenterology 1989;96:11-7.
8. Knight LC, Parkman HP, Brown KL, Miller MA, Trate DC, Maurer AH, Fisher RS Delayed gastric emptying and decreased antral contractility in normal premenopausal women compared with men. Am J Gastroenterol 1997;92:968-75.

9. Kumar D. In vitro inhibitory effect of progesterone on extrauterine human smooth muscle. Am J Obstet Gynecol 1962;84:1300-4.

10. Madsen JL. Effects of gender, age and body mass index on gastrointestinal transit times. Dig Dis Sci 1992;37:1548-53.

11. McCallum RW, Gull BB, Lange R, Planley M, Glass EE, Greenfield DG. Definition of a gastric emptying abnormality in patients with anorexia nervosa. Dig Dis Sci 1985;30:713-22.

12. Read NW, Houghton LA. Physiology of gastric emptying and pathophysiology of gastroparesis. Gastroenterol Clin North Am 1989;18:359-73.

13. Shay S, Eggli D, McDonald C, Johnson L. Gastric emptying of solid food in patients with gastroesophageal reflux. Gastroenterology 1987;92:459-65.

14. Urbain J-LC, Charkes ND. Recent advances in gastric emptying scintigraphy. Sem Nucl Med 1995;25:318-25.

15. Wald A, van Thiel DH, Hoechstetter L. Gastrointestinal transit: the effect of the menstrual cycle. Gastroenterology 1981;80:1497-500.

Recebido para publicação em 16/6/1999. Aprovado para publicação em 27/8/1999. 